\title{
Uber eine Verlandungsfolge weichsel-interstadialen Alters in Harksheide bei Hamburg
}

\author{
Von R. HAllik, Hamburg. \\ Mit $5 \mathrm{Abb}$. und 2 Tabellen. \\ Mitteilung Nr. 18 aus dem Geologischen Landesamt Hamburg.
}

$\mathrm{Zus}$ ammenfass ung. Ein aufgrund der Lagerungsverhältnisse - im Liegenden Eem, im Hangenden Solifluktionsschutt - - in ein Weichsel-Interstadial eingestuftes Vorkommen organogener Bildungen wird beschrieben. Es läßt sich ein vollständiger Verlandungszyklus feststellen. Dieser Umstand und das pollenstatistische Bild der Vegetationsentwicklung zeigen auch unabhängig von den Lagerungsverhältnissen, daß es sich um Bildungen einer selbständigen, in sich abgeschlossenen Warmphase handelt. Es ergibt sich das vollständige Bild eines WeichselInterstadials von der beginnenden Einwanderung der Vegetation bis zu den ersten Anzeichen einer neuerlichen Lichtung der Bewaldung. Das gewonnene Bild der Vegetationsentwicklung vermittelt eine Vorstellung von der Amplitude der Klimaoszillation. D i e s e hat höchstens das Ausmaß der Allerödschwankung erreicht.

Ein zweites unvollständiges Profil ist nach den Lagerungsverhältnissen und dem Pollendiagramm mit dem ersteren gut vergleichbar und daher ebenfalls als Weichsel-Interstadial anzusehen.

$\mathrm{S} \mathrm{u} \mathrm{m} \mathrm{m} \mathrm{a} \mathrm{r} \mathrm{y} \mathrm{.} \mathrm{Near} \mathrm{Harksheide} \mathrm{north} \mathrm{of} \mathrm{Hamburg} \mathrm{in} \mathrm{a} \mathrm{boring} \mathrm{organogenous} \mathrm{lake-sediments}$ and peat were found which in accordance with their stratigraphical position in the profile must have developed during a warmer period of the Last Glaciation - i. e. the WeichselGlaciation. There fore these Weichsel-Interstadial deposits are bound at the bottom by organogenous layers of the Eem-Interglacial. They are separated from these by a sand layer which is regarded as periglacial solifluction material. The boundary at the top is also formed by sand which corresponds with the solifluction deposits of the Weichsel-Glacial known in this area.

Two facts substantiate the age and give proof of the almost undisturbed conservation of this interstadial profile: 1.) there is a younger complete organogenous series; 2.) the development of vegetation shows differences in climate ranging from cool to a moderate warming-up and again to a renewed cooling off. The amplitude of the climatic oscillation has hardly reached the extend of the Alleröd-Interstadial.

The pollen-statistical diagram is neither comparable with the early Weichsel-Glacial nor with the Late Glacial.

Die Kenntnis von den organogenen Bildungen eines oder mehrerer Interstadiale der Weichselvereisung ist zwar nicht sehr jung, trotzdem aber mehr als lückenhaft. Es bestehen sogar - vor allem in Süddeutschland - Meinungsverschiedenheiten darüber, ob während der letzten Vereisung überhaupt eine oder mehrere wärmere Perioden vorhanden waren, die eine Rückwanderung der Vegetation und die Bildung organogener Sedimente ermöglicht haben hönnten (Büdel 1950, Brunnacker 1953, Graul 1953, SChaeFER 1953, FREISING 1954).

Im Bereich der nordeuropäischen Vereisung scheinen die Verhältnisse - vielleicht dank der Großräumigkeit des glazialen Geschehens - etwas klarer zu sein. Obgleich auch hier das Vorhandensein sicher nachgewiesener Interstadiale, bekundet durch organogene Bildungen, in vielen Fällen angezweifelt wird. Das bezieht sich in erster Linie auf ältere Angaben, z. B. auf das sogenannte Masurische Interstadial oder auch das Interstadial von Smidstrup in Dänemark. Eine zusammenfassende Darstellung des bisher über die Interstadiale der Weichsel-Eiszeit Bekannten hat zuletzt Woldstedt (1954) gegeben. Von den Funden jüngeren Datums wäre als erstes Interstadial das von Hengelo-Waarbeek in Holland (v. D. VleRK \& FLORSCHütz 1950) zu nennen. Besonders in den letzten Jahren haben sich dann Angaben ïber ähnliche Bildungen auch in Nordwestdeutschland gehäuft. Von SElle $(1952,1954)$ liegen Beiträge aus Nedden-Averbergen und Orel sowie aus Vechelde vor; Ditrmen (1954) beschreibt ein Vorkommen 
aus Nordfriesland, und Guenther (1951) fand Anzeichen einer wärmeren Phase mit entsprechender Fauna bei Preetz in Schleswig-Holstein, die jünger als die M-Moräne und älter als die letzten Vorstöße der Weichselvereisung in Schleswig-Holstein ist. Auf der Tagung der Deutschen Quartärvereinigung 1954 berichtete Kolumbe über ein Vorkommen weichsel-interstadialer organogener Bildungen aus Loopstedt bei Schleswig.

Mit Ausnahme der Vorkommen von Hengelo-Waarbeek und Vechelde kann man aber bei den genannten noch von keinem schlüssigen Nachweis eines Interstadials sprechen. Dazu wäre erforderlich, daß neben den Lagerungsverhältnissen die Entwicklung der organogenen Sedimente und der sedentären Bildungen sowie der Vegetation einen in sich abgeschlossenen vollständigen Zyklus aufzuweisen hätten. Beim Vorkommen von Vechelde erscheint das Fehlen einer limnischen Phase eigenartig. Doch kann das mit der besonderen Lage dieses Bohrpunktes in Zusammenhang stehen. Die ältesten bekannt gewordenen Funde in Schleswig-Holstein stammen von P. FrIEdRICH (1905, 1909), MenZel (1910) und STruck (1901, 1909 ${ }^{1}$ ).

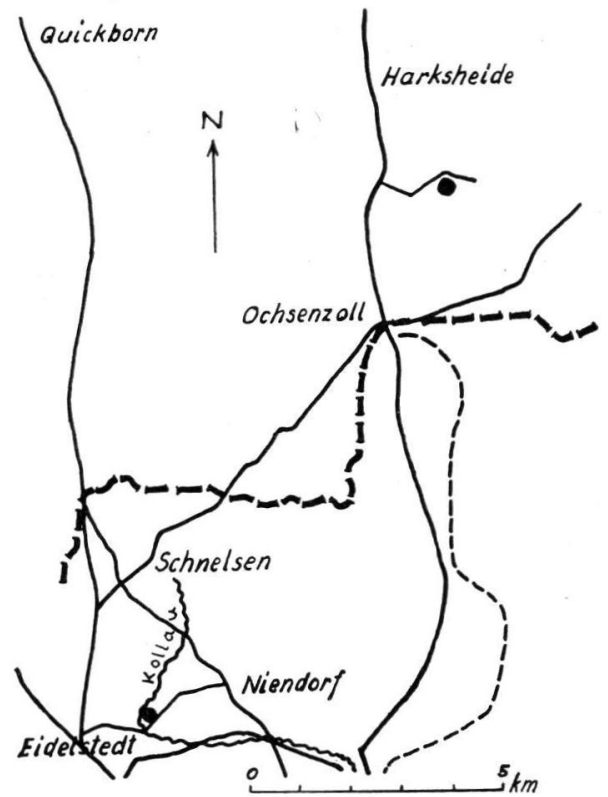

Abb. 1. Lage der Interstadial-Vorkommen.

In der vorliegenden Mitteilung sollen zwei weitere Vorkommen organogener Bildungen vorgelegt werden, deren Alter als Weichsel-Interstadial gesichert erscheint. Von diesen ist das erstere durch seinen Aufbau in besonderem Maße geeignet, eine grundlegende Stellung unter den bisher bekannt gewordenen Interstadialvorkommen einzunehmen. Es handelt sich um zwei Lokalitäten, in denen in Verbindung mit organogenen Junginterglazialvorkommen (Eem) - im Hangenden derselben - weitere limnische und telmatische Schichten angetroffen wurden, die noch nicht dem Spät- oder Postglazial angehören können. Es sind dieses: die Siedlung Falkenberg bei Harksheide nördlich von Hamburg und die Siedlung Waldfrieden an der Kollau bei Hamburg-Niendorf (Abb. 1).

Bei Harksheide wurden vom Geologischen Landesamt Hamburg zwei Bohrungen in einem Abstand von $1 \mathrm{~m}$ ausgeführt, die beide im Profil als auch im Pollendiagramm gut übereinstimmen. Dabei wurden unter Beachtung aller Vorsichtsmaßnahmen und

1) Die Hinweise auf diese Veröffentlichungen verdanke ich Herrn Prof. Dr. K. Gripp. 
unter ständiger Kontrolle fortlaufend gekernte Proben entnommen. Das erbohrte Profil hat folgenden Aufbau:

1. 0.00 bis $0.60 \mathrm{~m}$ Sand, bräunlich;

2. $\quad$ $1.75 \mathrm{~m}$ Sand, gelblich, z. T. mit Kies;

3. $\quad 1.95 \mathrm{~m}$ Sphagnum-Torf, Eriophorum, z. T. Braunmoos, oberste Probe mit sehr feinem Sand;

4. $\quad \quad 2.00 \mathrm{~m}$ Übergangstorf;

5. $\quad 2.05 \mathrm{~m}$ Mesotropher? Flachmoortorf, Sphagnum, Braunmoose, CarexRadizellen, Comarum, Menyanthes;

6. $\quad 2.10 \mathrm{~m}$ Nasse Lage, Myriophyllum;

7. $\quad 2.20 \mathrm{~m}$ Sphagnum-Torf, Braunmoos, Eriophorum, Scheuchzeria;

8. $\quad 2.30 \mathrm{~m}$ Mesotropher? Übergang, Sphagnum, Braunmoos, Typhaceae;

9. $2.35 \mathrm{~m}$ Limnisch-telmatischer Übergang, Nymphaeaceae, Typhaceae, Carex-Radizellen, Comarum, Menyanthes;

10. $\quad 2.60 \mathrm{~m}$ Grobdetritus-Gyttja, oben wenig Sand, nach unten zunehmend; Nymphaeaceae, Typhaceae, Comarum, Menyanthes, Myriophyllum, Pediastrum (im unteren Teil);

11. $\quad 3.00 \mathrm{~m}$ Sand, fein-mittel;

12. $\quad 3.20 \mathrm{~m}$ Sand, braun, aufgearbeiteter Torf, Feinkies;

13. $\quad 3.65 \mathrm{~m}$ Bruchwaldtorf;

14. $\quad \quad 3.75 \mathrm{~m}$ Thelypteris-Torf;

15. $\quad 6.85 \mathrm{~m}$ Gyttja, stark ockerhaltig, von 4.20-4.60 mit Konkretionen;

16. $\quad 7.30 \mathrm{~m}$ Sand, Kies.

Hier zeigen sich zwei selbständige Verlandungsfolgen, von denen auch die obere einen vollständigen, abgeschlossenen Zyklus aufweist, der allerdings einmal durch eine Rekurrenz unterbrochen wird, die - wie aus dem Pollendiagramm (Abb. 3) hervorgeht - klimatisch bedingt $\mathrm{zu}$ sein scheint.

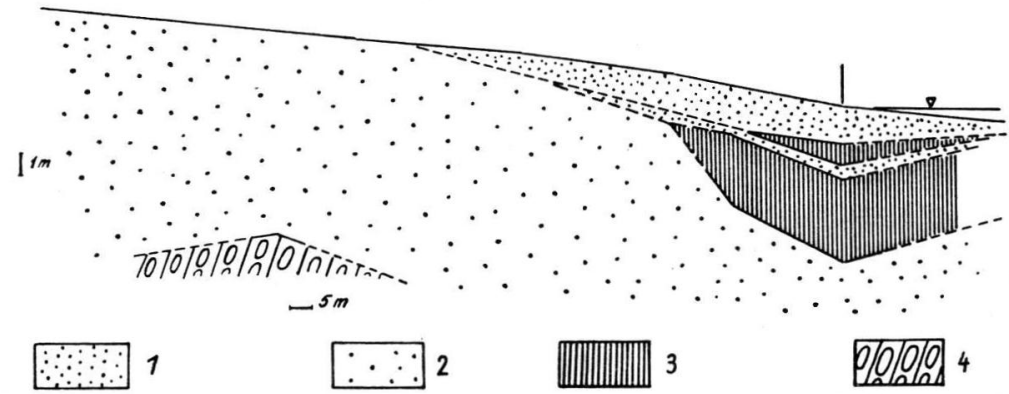

Abb. 2. Durch Bohrungen ermitteltes Profil des Vorkommens bei Harksheide. 1 = Periglaziale Fließerde, 2 = Sande des Warthe-Stadiums, $3=$ Organogene Bildungen, $4=$ Geschiebelehm.

Eine eingehendere Erörterung der Lagerungsverhältnisse ist für die Beurteilung der Bedeutung dieses Profils erforderlich. Wie aus Abb. 2 ersichtlich, liegt das Vorkommen im Untergrund einer kleinen Senke, deren tiefste Stelle von einem kleinen Teich eingenommen wird. Das bearbeitete Profil ist unmittelbar am Teichrand (ca. $+32.5 \mathrm{~m} \mathrm{NN}$ ) entnommen worden. Nach den Seiten steigt das Gelände auf einer Strecke von ca. $160 \mathrm{~m}$ um $4.5 \mathrm{~m}$ an. Es liegen hier also für die ungestörte Erhaltung der organogenen Bildungen besonders günstige Verhältnisse vor, $\mathrm{da}$ ja eine derartige kleine Senke eher einen lokalen Sedimentationsraum als ein Erosionsgebiet darstellen wird.

Die heutige Morphologie wird allerdings nicht dem Zustand während der Bildung der interglazialen und interstadialen Schichten entsprechen. Ein gewisses Ausmaß einer nachträglichen Sackung der organogenen Bildungen muß unbedingt berücksichtigt wer- 
den. Periglaziale Bodenbewegungen, die zu einer Zerstörung des obersten Teiles der liegenden Interglazial-Ablagerungen geführt haben, zeigen sich darin, daß die Interglazialfolge schon mit dem Beginn des Pinus-Anstieges im pollenstatistischen Diagramm endet. Das Frühglazial fehlt auch hier, wie das meistens der Fall ist. Außerdem ist an der Basis der oberen Verlandungsserie eine wenige Zentimeter dicke Schicht zusammengeschwemmten tonig-sandigen Materials mit der Pollenführung des Interglazials zu finden.

Im Hangenden wird die obere Verlandungsfolge durch teilweise humose Sande mit einer gewissen Kieskomponente abgeschlossen. Auch hier handelt es sich wenigstens zum Teil um periglaziale Umlagerungserscheinungen, durch die ein Teil der organogenen Bildungen zerstört sein könnte. Es liegt hier demnach eine organogene Serie vor, die im Liegenden von eem-interglazialen Bildungen (von diesen durch eine Sandlage, deren Entstehung periglazialen Bodenbewegungen zuzuschreiben wäre) und im Hangenden

Tabelle 1: Weitere, im Diagramm nicht aufgeführte Pollen- und Sporentypen des Profils bəi Harksheide (absolute Zahlen).

\begin{tabular}{|c|c|c|c|c|c|c|c|c|c|c|c|c|c|c|c|c|c|c|c|c|c|}
\hline $\begin{array}{l}\dot{\vec{z}} \\
\dot{0} \\
\dot{0} \\
\dot{a} \\
\end{array}$ & 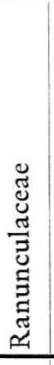 & 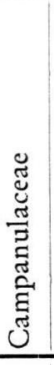 & 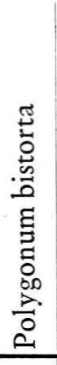 & 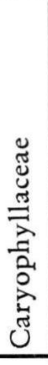 & 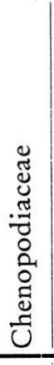 & 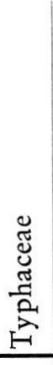 & 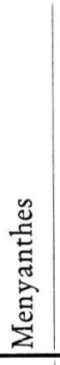 & 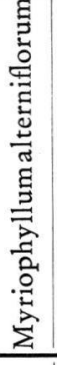 & 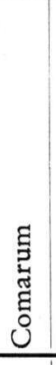 & 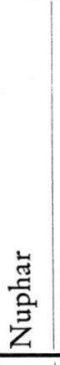 & 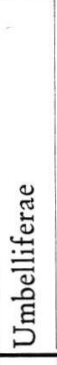 & 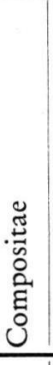 & $\begin{array}{l}\tilde{J} \\
.0 \\
\tilde{\tilde{J}} \\
\tilde{\omega} \\
\ddot{u} \\
\dot{u}\end{array}$ & 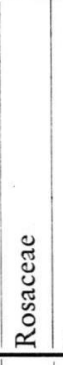 & 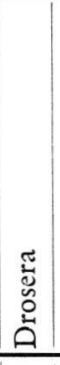 & 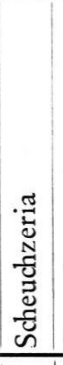 & 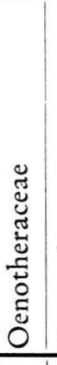 & 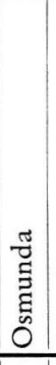 & 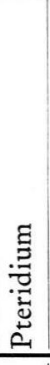 & 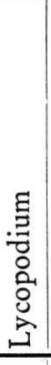 & 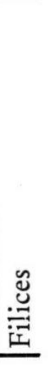 \\
\hline 8 & & & & & & & & & & & & & & & & & & & 3 & 1 & \\
\hline 9 & & & & & & & & & & & & & & & & & & & 1 & & 3 \\
\hline $9 a$ & & 1 & & & & & & & & & & & & & & & & & & & \\
\hline 10 & & & & & & & & & & & & & & 1 & & & & & & & \\
\hline 11 & 1 & & & & & & & & & & & & & & & & & & & & 2 \\
\hline 12 & & & & & & & 1 & & & & 1 & & & 1 & 1 & 12 & & & & & \\
\hline 13 & & & & & & & 1 & & 1 & & & & & & & & & & & & 1 \\
\hline 14 & & & & 1 & & & & & & & & & & 1 & & & & & & & \\
\hline 15 & & 1 & & & & & & 1 & & & & 1 & & & & & & & & 2 & 1 \\
\hline 16 & & & & & & & & & & & 1 & 2 & & 1 & & & & & & & \\
\hline 17 & & & & & & 1 & & & & & & 1 & & & & & & & 1 & & 4 \\
\hline 18 & & & & 2 & & 1 & & & & & 1 & & 1 & 1 & & & & & & & \\
\hline 19 & & & & & & 2 & 2 & & & & 2 & 1 & 1 & & & & & 1 & 3 & & 3 \\
\hline 20 & 1 & & & & & 2 & 1 & & 1 & 1 & 3 & & & & & & & & & & \\
\hline 21 & & & & 2 & & 3 & 1 & & 1 & & 2 & & & 1 & & & 1 & & & & 1 \\
\hline 22 & 1 & & & 3 & & 8 & & & 1 & 1 & 2 & 2 & & 4 & & & & & & & 5 \\
\hline 23 & 2 & & & 3 & 1 & 11 & & 4 & 3 & & 1 & 2 & & 2 & & & & & 1 & & 2 \\
\hline 24 & & 1 & 1 & 5 & 1 & 6 & & 3 & & & & 3 & & 4 & & & & & & 1 & 2 \\
\hline
\end{tabular}


durch ebenfalls periglazialen Solifluktionsschutt begrenzt wird. Damit ergibt sich eine Altersstellung, wonach diese Serie jünger als das Eem einschließlich des Frühglazials und älter als endweichseleiszeitlich ist.

Wie bereits angedeutet, kann dieser Beweis für ein weichsel-interstadiales Alter allein noch nicht befriedigen. Ein Vergleich des Pollendiagramms von Harksheide mit denen des Weichselfrühglazials und des Spätglazials kann erst die Altersdeutung der Vollständigkeit näherbringen.

Um ein Weichselfrühglazial (HaLLIK 1952) kann es sich nicht handeln, denn 1.) liegt am Ende des Eem-Interglazials in diesem Profil ein eindeutiger Stillstand der sedentären Bildungen, ja sogar eine teilweise Zerstörung derselben vor; 2.) beginnt der neue Sedimentationszyklus mit einer Gyttja, die besonders im unteren Teil reich an minerogenen Beimengungen ist; sie ist demnach in einer Zeit dürftiger Vegetationsbedeckung entstanden; in die gleiche Richtung weist auch der hohe relative Anteil der NBP im Pollendiagramm (Abb. 3); 3.) ist im Pollendiagramm, wenn auch nicht vollständig, so doch zur Genüge angedeutet, durch die Entwicklung der Vegetation ein Klimaverlauf von kühl über ein sehr schwach ausgeprägtes Optimum zu einer erneuten Abkühlung abzulesen.

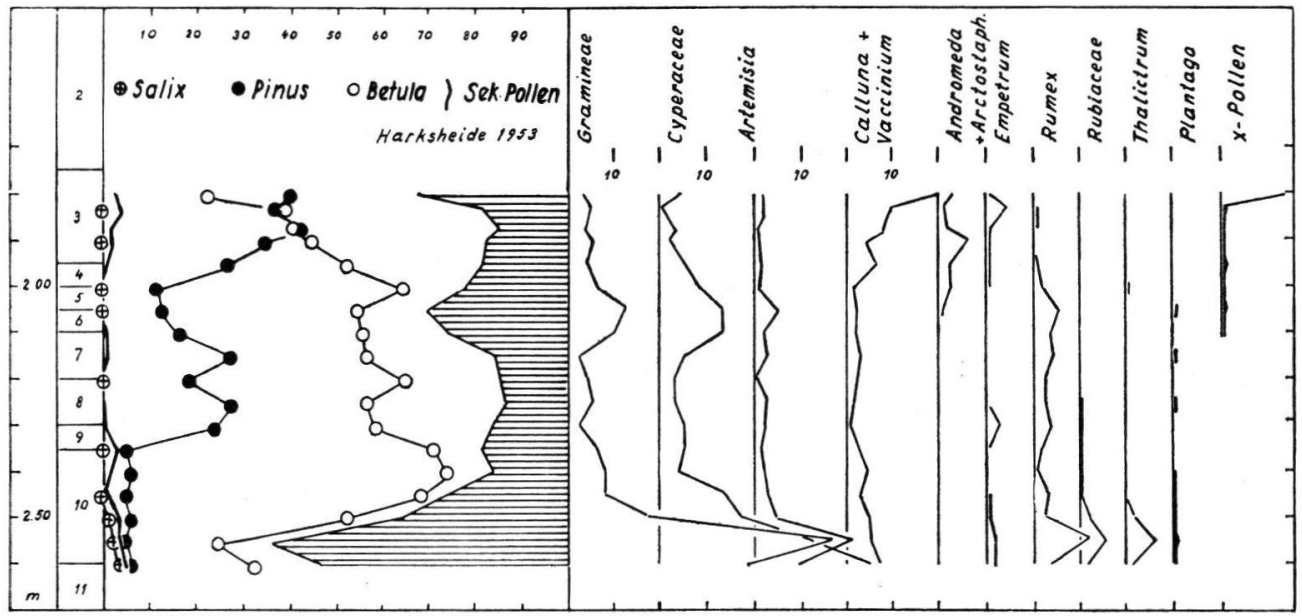

Abb. 3. Pollendiagramm des Interstadial-Vorkommens Siedlung Falkenberg bei Harksheide. (Unter Benutzung der allgemein üblichen Signaturen).

Die Unterschiede gegenüber den zahlreichen bekannten Diagrammen des Spätgla-zials sind ebenfalls recht bezeichnend: Wohl am auffälligsten ist das sehr schnelle, fast vollständige Verschwinden von Salix. Allochthoner und wahrscheinlich auf Ferntransport zurückzuführender Pollen (im Diagramm dick ausgezogene Linie) ist fast geschlossen durch das ganze Profil vertreten. Bezeichnenderweise fehlen ausgesprochen tertiäre Typen. Unmittelbar auf eine waldlose bis waldarme Phase folgt ein ausgesprochenes Betula-Maximum. Es wäre durchaus anzunehmen, daß die geringen Pollenfrequenzen von Pinus (um 5\%) ebenso wie Picea, Alnus und andere in dieser Schicht nur aus dem zerstörten Teil des liegenden Eem-Interglazials stammen. Dieser Úbergang von der waldarmen Phase zur Betula-Phase, die an den ersten Abschnitt des Alleröd-Interstadials erinnern könnte, tritt hier im Gegensatz zum Spätglazial sehr abrupt ein. Daß es sich nicht um das Alleröd-Interstadial handeln kann, geht ferner daraus hervor, daß der im oberen Teil des Profils auftretende Pinus-Anstieg dann bereits der präborealen Klimaperiode des beginnenden Postglazials angehören müßte, wofür alle sonstigen Anzeichen fehlen. Gänzlich unverständlich wäre dann schon das Auftreten des Picea- 
Pollens; einerlei, ob dieser als ferntransportiert oder als sekundär umgelagert anzusehen wäre.

Wenn überhaupt bei dem geringen, bis jetzt vorliegenden Material ein Versuch unternommen werden kann, das vorliegende Profil mit anderen als weichsel-interstadial erkannten zu vergleichen, so wäre dies am ehesten mit dem von Vechelde (SELLE 1954) möglich. Doch zeigen sich auch hier insofern Schwierigkeiten, als in Vechelde ein dreimaliges Pinus-Maximum zu erkennen ist, während in Harksheide nur zwei zu finden sind. Es ist durchaus möglich, daß in Harksheide der oberste Teil der organogenen Bildungen - die dem dritten Pinus-Maximum in Vechelde entsprochen hätten durch die Einwirkung periglazialer Bodenbewegungen zerstört worden ist.

In beiden Fällen zeigen sich aber im Hangenden der Serie Anzeichen für ein Kühlerwerden des Klimas und ungefähr in der Mitte des Profils ein durch die Zunahme des relativen Anteils der NBP angedeuteter Rückgang der an sich wohl lockeren Bewaldung (Maxima der Gramineen, Cyperaceen, Artemisia, Rumex, Plantago). Im Profil Harksheide beginnt an dieser Stelle ein Pollentyp aufzutreten, der im Frühglazialprofil von Lüneburg (HALLIK 1952) als „Rhamnus frangula?" bezeichnet war (im vorliegenden Profil x-Pollen). Diese Bestimmung wird wohl falsch sein, so daß dieser Pollentyp für eine Deutung der Vegetationsverhältnisse ohne Bedeutung bliebe, wenn sich nicht gerade in dem Profil aus Lüneburg gezeigt hätte, daß es sich um ein Vegetationselement aus dem Bereich der subarktischen Waldgrenze zu handeln scheint. Hier erreicht derselbe sein maximales Vorkommen in der obersten Probe.

Der Anstieg der NBP-Kurve im obersten Teil des Diagramms beruht zu einem gewissen Teil auf•der Zunahme der Ericaceen. Das kann die Folge von deren lokaler Ausbreitung auf dem Sphagnum-Moor sein und wäre somit noch nicht beweisend. Unterstützt wird die Annahme einer fortschreitenden Lichtung der Bewaldung durch eine gleichzeitige Zunahme des sekundären Pollens - hier wird wiederum ein Teil des Pinus-Pollens dazu zu rechnen sein. Als weiteres Anzeichen eines Rückganges der Vegetationsbedeckung und gleichzeitig als Erklärung für das Auftreten des sekundären Pollens findet sich im obersten Teil des Profils die bereits erwähnte Sandbeimengung.

Außer dem im Diagramm dargestellten Baumpollen fanden sich - mehr oder weniger vereinzelt auftretend - Pollentypen thermophiler Flemente. Diese sind im Diagrammbild (Abb. 3) als sekundärer und ferntransportierter Pollen mit der dick

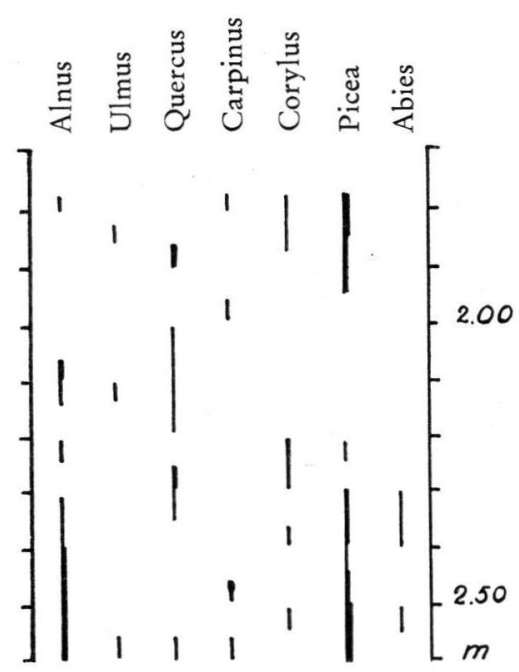

Abb. 4. Verteilung des sekundären Pollens im Profil bei Harksheide. 
ausgezogenen Linie gekennzeichnet. Deren Verteilung im Einzelnen ist in Abb. 4 dargestellt. Im unteren Teil des Profils, in der Gyttja, ist ihr Auftreten als umgelagerter Pollen verständlich, zumal ja die minerogene Komponente auf die Möglichkeit der Einschwemmung hindeutet. Dasselbe trifft auch für den obersten Teil des Profils zu. In den rein autochthonen Schichten muß der vereinzelt auftretende Pollen als Ferntransport angesehen werden, obgleich eine brauchbare Deutung bei dieser eigenartigen $\mathrm{Zu}$ sammensetzung nicht ohne weiteres zu finden ist.

Erwähnenswert ist auch das relativ häufige Auftreten einer abgewandelten Form des Empetrum-Pollens im obersten Teil des Diagramms. Dieser tritt als Dyade auf, bei der die für die Ericales typischen Falten noch vorhanden sind. Durch einen Vergleich mit rezentem Material konnten die Dyaden als Empetrum identifiziert werden. Eine eingehende Bearbeitung dieser eigenartigen Formen hat Herr Dr. G. Endtman iibernommen. Weitere, in den Diagrammen nicht aufgeführte Pollen- und Sporentypen sind in den beiden Tabellen zusammengefaßt. Auch diese enthalten $z$. T. sicher sekundäres Material.

Eine Gliederung des vorliegenden Interstadial-Profils in Phasen der Vegetationsentwicklung vorzunehmen, stößt auf einige Schwierigkeiten. Vor allem entstehen Unsicherheiten in der Bewertung der Pinus-Verteilung. Die klimatischen Verhältnisse werden, bedingt durch die wohl nicht allzu ferne Inlandeismasse, vielleicht nicht mit denen des Postglazials in Nordfinnland direkt vergleichbar sein. Zudem ist die Frage der vorherrschenden Windrichtungen während des Weichselglazials nicht gelöst. Sie ist aber im Hinblick auf den Ferntransport von Pollen von Bedeutung. Man wird aber doch die von AARIO (1944) getroffenen pollenanalytischen Feststellungen in den waldlosen bis waldarmen Gebieten Nordfinnlands nicht übersehen dürfen, wenn zur Frage der Pinus-Ausbreitung in Schleswig-Holstein während der Interstadialzeit Stellung genommen werden sollte. Die Beantwortung dieser Frage wäre zunächst noch zurückzustellen.

Es wäre in diesem Zusammenhang nur auf den eigenartigen Befund hinzuweisen, den Guenther (1951) in seiner Beschreibung des Elchfundes bei Preetz in SchleswigHolstein gibt. Wenn auch nur eine mangelhafte pollenanalytische Bearbeitung vorliegt, so ist trotzdem auffallend, daß bei Preetz Pinus-Pollen überhaupt nicht gefunden wurde, während dieser in den von Ditrmer (1954) aus Nordfriesland beschriebenen

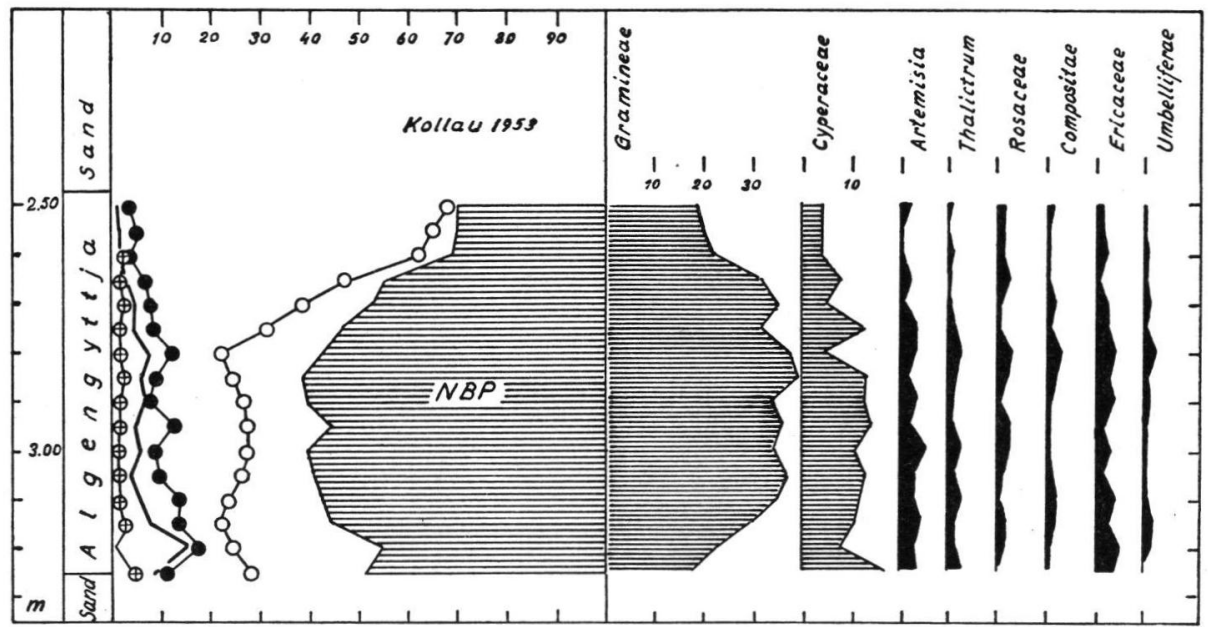

Abb. 5. Pollendiagramm des Interstadial-Vorkommens Siedlung Waldfrieden an der Kollau bei Hamburg-Niendorf (Signaturen wie bei Abb. 3). 
Interstadial-Vorkommen angetroffen wurde. Wenn man mit DückER (Vortrag auf der Tagung der Deutschen Quartärvereinigung 1954) für die Weichselglazialzeit vorwiegend östliche oder nordöstliche Windrichtungen annehmen wollte, könnte der Befund von Guenther verständlich erscheinen. Dabei muß allerdings auch die Frage offenbleiben, ob die vorherrschenden Windrichtungen während der Interstadialzeit dieselben gewesen sein müssen wie zur Zeit einer hochglazialen Periode.

Diese Vergleiche setzen eine Gleichaltrigkeit der interstadialen Bildungen von Vechelde, Harksheide, Nordfriesland und Preetz voraus. Diese ist zwar keineswegs bewiesen, jedoch könnte ihr ein gewisser Grad der Wahrscheinlichkeit zugebilligt werden.

Das Vorkommen an der Kollau in Hamburg besteht - sedimentologisch gesehen aus einer grauen, feingeschichteten Algengyttja, die weit überwiegend aus Schalen von Süßwasserdiatomeen und Pediastrum-Arten besteht. Auch hier finden sich im Liegenden organogene Bildungen des Eem-Interglazials. Es folgt wieder ein z. T. grobkiesiger Sand, weiter die Algengyttja mit einer Mächtigkeit von $0.75 \mathrm{~m}$ und schließlich ein kiesiger, schwach toniger Sand als Solifluktionsschutt. Das Pollendiagramm (Abb. 5) ist gut mit dem von Harksheide vergleichbar. Es stellt allerdings nur den untersten waldarmen Teil mit allen hierfür typischen Merkmalen dar. Lagerungsverhältnisse und Vergleichbarkeit der Vegetationsentwicklung mit dem vollständigeren Profil von Harksheide ermöglichen es, auch dieses Vorkommen als Weichsel-Interstadial anzusehen.

Tabelle 2: Weitere, im Diagramm nicht aufgeführte Pollen- und Sporentypen des Profils an der Kollau (absolute Zahlen).

\begin{tabular}{|c|c|c|c|c|c|c|c|c|c|c|c|c|c|c|c|c|c|c|c|}
\hline 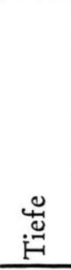 & 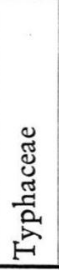 & 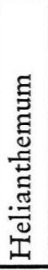 & 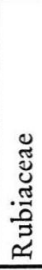 & 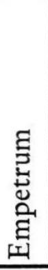 & 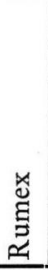 & 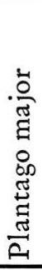 & 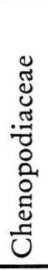 & 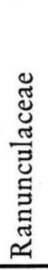 & 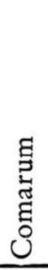 & 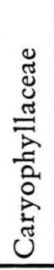 & 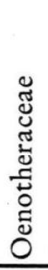 & 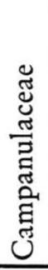 & 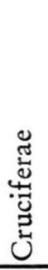 & 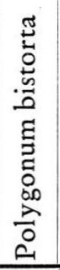 & 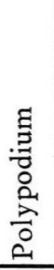 & 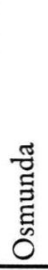 & 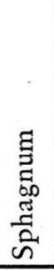 & 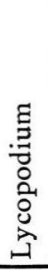 & 氖 \\
\hline 2.50 & 8 & 1 & 1 & 1 & & & & & & & & & & & & & 5 & & 3 \\
\hline 2.55 & 2 & & 1 & & 1 & 1 & 1 & & & & & & & & 1 & & 2 & & \\
\hline 2.60 & 2 & & & & & & & & & & & & & & 2 & & 6 & & \\
\hline 2.65 & 13 & & 1 & & 1 & & 1 & 3 & & & & & & & & & 19 & 1 & 1 \\
\hline 2.70 & 11 & & 2 & & 2 & & & & 1 & 1 & & & & & & & 25 & & 4 \\
\hline 2.75 & 6 & & 3 & 1 & 3 & & & & & & & & & & & & 42 & & 1 \\
\hline 2.80 & 16 & & 2 & 2 & & 1 & & & & & 1 & & & & & & 13 & & 4 \\
\hline 2.85 & 39 & & & & & 1 & & & & & & & & & & 3 & 39 & & 6 \\
\hline 2.90 & 27 & & 3 & 7 & 1 & & 1 & & 1 & 1 & & 1 & & & & & 30 & 1 & 3 \\
\hline 2.95 & 37 & & 1 & 1 & 1 & & 3 & & & 1 & & & 1 & 1 & 1 & 1 & 21 & & 5 \\
\hline 3.00 & 23 & & 1 & & & & 1 & & 2 & 2 & & & & & & & 29 & & 4 \\
\hline 3.05 & 42 & & 1 & 1 & & & 2 & & & 3 & & 1 & & & 1 & 1 & 34 & & \\
\hline 3.10 & 60 & & 1 & 1 & & & & 1 & 2 & & & & & & & & 34 & & 3 \\
\hline 3.15 & 36 & & 3 & 1 & 1 & & 1 & & 2 & 4 & & & & 1 & & 2 & 41 & & 4 \\
\hline 3.20 & 25 & & 2 & 1 & 1 & 1 & & 1 & & & 1 & 1 & & & & 2 & 23 & & 4 \\
\hline 3.25 & 15 & & 7 & 2 & 1 & 1 & & & & 2 & & & & & & & 42 & & 9 \\
\hline
\end{tabular}


Abschließend wäre die Möglichkeit zu erwähnen, daß die als Herningtypus (JESSEN \& Milthers 1928) bezeichneten Junginterglazial-Profile auf zweierlei Entstehungsmöglichkeiten zurückzuführen sein könnten. Zu einem Teil wird es sich sicherlich um Umlagerungserscheinungen handeln, wie diese von THOMson (1951) nachgewiesen worden sind. Ein gewisser Teil derselben könnte sich aber nach erneuter Überprüfung vielleicht doch als den Profilen von Harksheide, Vechelde und ähnlichen vergleichbar herausstellen.

\section{Literaturverzeichnis}

Aario, L.: Uber die pollenanalytischen Methoden zur Untersuchung von Waldgrenzen. - Geol. Fören. i. Stockholm Förhandl. 66, S. 337-354, Stockholm 1944.

BRUNNACKER, K.: Der würmeiszeitliche Löß in Südbayern. - Geologica Bavarica 19, 1953.

BüDEL, J.: Die Klimaphasen der Würmeiszeit. - Die Naturwissenschaften 37, 1950.

DitTMER, E.: Interstadiale Torfe in würmeiszeitlichen Schmelzwassersanden Nordfrieslands. Eiszeitalter und Gegenwart 4/5, 1954

Freising, H.: Gibt es in Hessen drei Würm-Lösse? - Jber. u. Mict. oberrhein. geol. Ver. 35, 1954.

Frenzel, B. \& Troll, C.: Die Vegetation des nördlichen Eurasiens während der letzten Eiszeit. - Eiszeitalter und Gegenwart 2, 1952.

Friedrich, P.: Die Grundmoräne und die jungglacialen Süßwasserablagerungen der Umgegend von Lübeck. - Mitt. geograph. Ges. u. naturhist. Mus. Lübeck, 2. Reihe, H. 20, 1905.

Friedrich, P.: Der geologische Aufbau der Stadt Lübeck und ihrer Umgebung. - Lübeck 1909.

GAMS, H.: Neue Beiträge zur Vegetations- und Klimageschichte der nord- und mitteleuropäischen Interglaziale. - Experientia 10, Basel 1954.

Graul, H. \& Schaefer, J.: Zur Gliederung der Würmeiszeit im Illergebiet. - Geologica Bavarica 18, 1953.

Guenther, E.: Ein eiszeitlicher Elch aus Preetz und die Frage eines Weichselinterstadials in Ost-Holstein. - Schr.naturw.Vers.Schleswig-Holstein 25 (Karl-Gripp-Festschrift), 1951.

HALLIK, R.: Ein „Weichsel-Frühglazial“-Profil in Lüneburg. - Eiszeitalter u. Gegenwart 2, 1952.

Jessen, K. \& Milters, V.: Stratigraphical and Paleontological Studies of Interglacial FreshWater Deposits in Jutland and Northwest-Germany. - Danmarks Geologiske Undersögelse, II Raekke Nr. 48, 1928.

Menzel, H.: Klimaänderungen und Binnenmollusken im nördlichen Deutschland seit der letzten Eiszeit. - Z. deutsch. geol. Ges. 62, Berlin 1911.

Selle, W.: Die Interstadiale der Weichselvereisung. - Eiszeitalter und Gegenwart 2, 1952. - Das Vechelder Interstadial. - Ebendort 4/5, 1954.

Struck, R.: Diluviale Schichten mit Süßwasserfauna an der Untertrave. - Jb. preuß. geol. Landesanst. 21, 1901.

Struck, R.: Ưbersicht der geologischen Verhältnisse Schleswig-Holsteins. - Festschrift zur Begrüßung des XVII. Deutschen Geographentages, Lübeck 1909.

Thomson, P. W.: Das Interglazial von Wallensen im Hils. - Eiszeitalter u. Gegenwart 1, 1951. VLERK, vAN DER, J. M. \& FlorschüTz, F.: Nederland in het Jjstijdvak. - Utrecht 1950.

Woldstedt, P.: Saaleeiszeit, Warthestadium und Weichseleiszeit in Norddeutschland. - Eiszeitalter und Gegenwart 4/5, Ohringen 1954.

Manuskr. eingeg. 5. 2. 1955.

Anschrift des Verf.: Dr. Rudolf Hallik, Geologisches Landesamt Hamburg, Hamburg 13, Rothenbaumchaussee $64 a$. 